\title{
Chirality Induced by Magnetoelectrolysis
}

\author{
I. Mogi ${ }^{a, *}, \mathrm{R} . \operatorname{AOGAKI}^{b}, \mathrm{R} . \mathrm{Morimoto}^{c}, \mathrm{~K} . \mathrm{WATANABE}^{a}$ \\ ${ }^{a}$ Institute for Materials Research, Tohoku University, Katahira, Aoba-ku, Sendai 980-8577, Japan \\ ${ }^{b}$ Polytechnic University, Sagamihara, Kanagawa 252-5196, Japan \\ ${ }^{c}$ Saitama Prefectural Okubo Water Filtration Plant, Saitama 338-0814, Japan
}

\begin{abstract}
Surface chiral formation of $\mathrm{Cu}$ films was investigated in the galvanostatic electrodeposition under a magnetic field of $5 \mathrm{~T}$ perpendicular to the electrode surface. The surface chirality was examined by electrochemical voltammograms of a chiral molecule of tartaric acid. The chiral induction depended on the magnetoelectrodeposition time, and this implied that the formation of the self-organized state of magnetohydrodynamic flows around the electrode is crucial for the chiral formation.
\end{abstract}

DOI: $10.12693 /$ APhysPolA.126.380

PACS: $47.35 . \mathrm{Tv}$

\section{Introduction}

Application of magnetic field to electrolysis involves various research aspects from viewpoints of electrochemistry, magnetohydrodynamics and astrophysical hydrodynamics. Particular concern has been devoted to the chirality induction on the surfaces of electrodeposits.

When magnetic field is imposed to an electrochemical cell, the Lorentz force acting on the faradaic currents causes flows around the electrode in the solutions. This is well known as the magnetohydrodynamic (MHD) effect [1]. Figure 1 shows two kinds of the MHD effects on an electrodeposition process [2], where the magnetic field is perpendicular to the electrode surface. Electrodeposition is a non-equilibrium phenomenon, which produces a lot of humps on the deposit surface. The Lorentz force acting on the currents around the humps causes microvortices near the surface. This is called the micro-MHD effect. Another MHD effect produces a macroscopic flow around the electrode edge, where the current direction is not parallel to the magnetic field, and then the macroscopic flow grows into the tornado-like rotating stream over the electrode surface. This is called the vertical MHD effect.

The micro-MHD vortices could produce chiral defects such as screw dislocations on the electrodeposit surfaces. As the adjoining flows never conflict with each other, both clockwise and anticlockwise vortices must coexist. Such a symmetrical vortex state leads to achiral surfaces. The influence of the vertical MHD flow on the microMHD vortices could break the symmetry of the microMHD vortices, inducing surface chirality [2].

We have tried to explore chiral surfaces of magnetoelectrodeposited (MED) metal films of $\mathrm{Ag}[3]$ and $\mathrm{Cu}[4,5]$. The MED films were employed as the electrodes, and the electrochemical reactions of chiral organic molecules

*corresponding author; e-mail: mogi@imr.tohoku.ac.jp

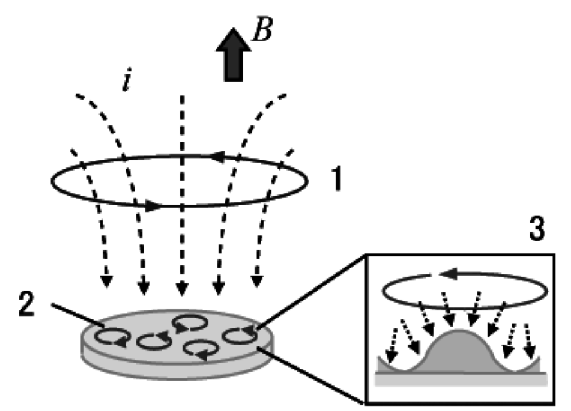

Fig. 1. MHD flows induced by magnetic field $\boldsymbol{B}$ perpendicular to the electrode surface and parallel to the faradaic currents $i$. 1 - the vertical MHD flow around the electrode edge, 2 - the micro-MHD vortices, 3 schematic of the micro-MHD vortex around the nonequilibrium fluctuation (humps) on the deposit surface.

such as amino acids and tartaric acid were examined by voltammetry. The chiral behaviour of the MED film electrodes appeared as the oxidation current difference between the enantiomers. The chiral behaviours of the MED films depended on the experimental conditions of the magnetoelectrodeposition, such as magnetic fields, electrode potentials and diameters. In this paper, we report the deposition time dependence on the chiral induction, to investigate the roles of micro-MHD and vertical MHD flows.

\section{Experimental}

$\mathrm{Cu}$ electrodeposition was conducted in a $50 \mathrm{mM}$ $\left(\mathrm{M}=\mathrm{mol} \cdot \mathrm{dm}^{-3}\right) \mathrm{CuSO}_{4}$ aqueous solution containing $0.5 \mathrm{M} \mathrm{H}_{2} \mathrm{SO}_{4}$. The $\mathrm{Cu}$ films were formed on a Pt electrode with a diameter of $1.6 \mathrm{~mm}$ by the galvanostatic electrodeposition at a current of $10 \mathrm{~mA} \cdot \mathrm{cm}^{-2}$. A magnetic field of $5 \mathrm{~T}$ was imposed perpendicularly to the electrode surface and parallel to the faradaic current.

In order to examine the chirality of the MED films, voltammograms of the enantiomers of tartaric acid (TA) 
were measured on the MED film electrodes. Voltammetry represents the measurements of faradaic currents $i$ of electrode reactions during the linear sweep of a potential $E$ of the working electrode.

\section{Results and discussion}

Figure 2 shows the voltammograms of the enantiomers of TA on the $\mathrm{Cu} 5 \mathrm{~T}$-film electrodes. The oxidation of both enantiomers occurs around $0.7 \mathrm{~V}$, but the oxidation currents are quite different; the peak current of L-TA is larger than that of D-TA. The voltammograms of the enantiomers coincided with each other on the 0T-film electrodes. These results indicate that the magnetoelectrodeposition induces the chiral structures on the the film surfaces and they can recognize the molecular chirality of TA.

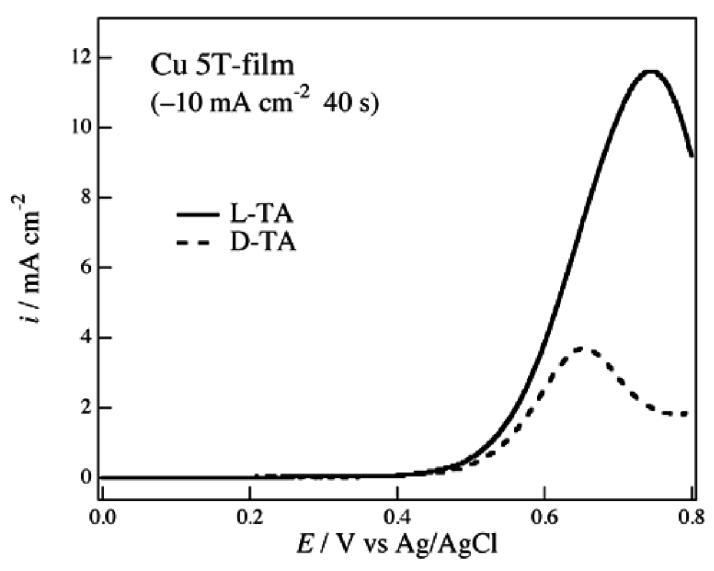

Fig. 2. Voltammograms of $20 \mathrm{mM} \mathrm{L}$ - and D-tartaric acid (TA) on the $\mathrm{Cu} 5 \mathrm{~T}$-film electrode in a $0.1 \mathrm{M} \mathrm{NaOH}$ aqueous solution. The 5T-film was prepared during deposition time of $40 \mathrm{~s}$. The potential sweep rate was $50 \mathrm{mV} \cdot \mathrm{s}^{-1}$

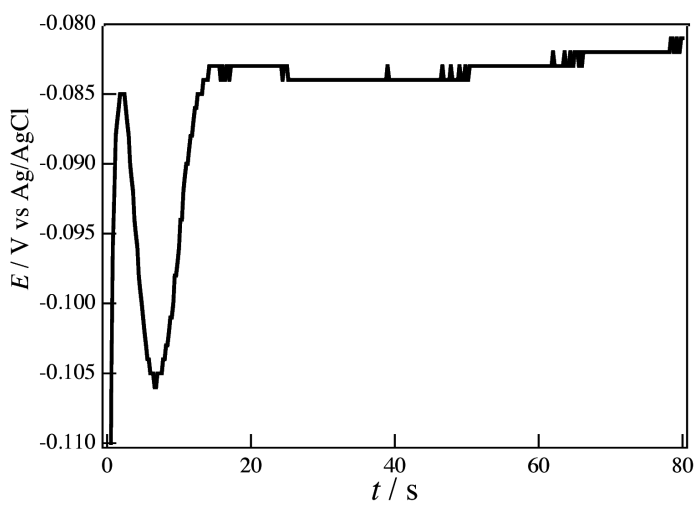

Fig. 3. Electrode potential $E$ vs time $t$ during the galvanostatic electrodeposition of $\mathrm{Cu}$ in a magnetic field of $5 \mathrm{~T}$. The electrodeposition current was $-10 \mathrm{~mA} \cdot \mathrm{cm}^{-2}$.

Figure 3 shows the electrode potential curve against time of up to $80 \mathrm{~s}$ during the electrodeposition at $5 \mathrm{~T}$.
The potential changes drastically at the initial $15 \mathrm{~s}$ and then stays nearly constant, forming a plateau around $-0.085 \mathrm{~V}$. In galvanostatic electrodeposition without magnetic field, the potential plateau means that a diffusion double layer has been formed near the electrode. On the other hands, under the magnetic fields, there exist the micro-MHD vortices and the vertical MHD flow around the electrode. Thus, the appearance of the potential plateau indicates the formation of self-organized state of these MHD flows, as shown Fig. 1. The network morphology on the surfaces of the 5T-films implied such a self-organized state [4].

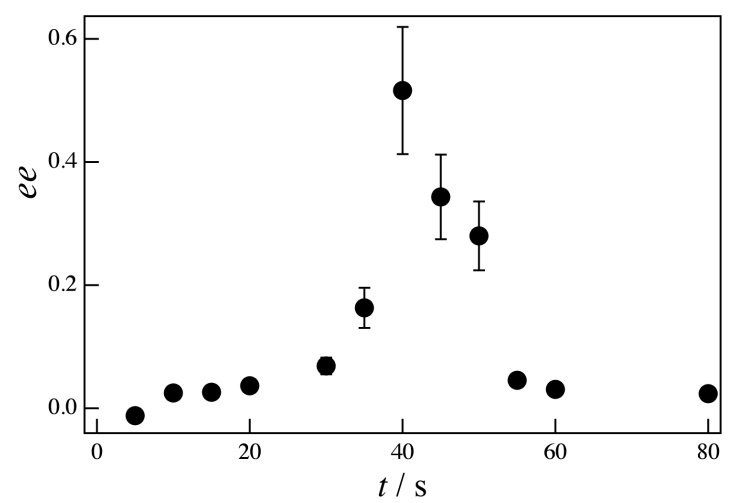

Fig. 4. Enantioexcess (ee) ratio vs magnetoelectrodeposition time $t$.

The chiral behaviors of the 5T-films were examined for various deposition times, and the results are shown in Fig. 4, where the enantioexcess (ee) ratios are plotted against the deposition times. The ee ratio in the voltammograms can be defined as $e e=\left(i_{p}^{L}-i_{p}^{D}\right) /\left(i_{p}^{L}+i_{p}^{D}\right)$, where $i_{p}^{L}$ and $i_{p}^{D}$ represent the peak currents of L- and D-TA, respectively. The chiral behavior appears at the deposition times from 30 to $50 \mathrm{~s}$, where the steady selforganized state of the MHD flows is formed, as shown in Fig. 3. At the deposition times shorter than $20 \mathrm{~s}$, the MHD flow state is transient, then the 5T-film is achiral. These facts imply that the self-organized state of the micro-MHD and vertical MHD flows is crucial for the formation of chiral surfaces. At the deposition times longer than $50 \mathrm{~s}$, with increasing the film thickness, the non-equiribrium fluctuation might disturb the formation of chiral structures.

\section{References}

[1] T.Z. Fahidy, J. Appl. Electrochem. 13, 553 (1983).

[2] R. Aogaki, R. Morimoto, in: Heat and Mass Transfer - Modeling and Simulation, ed. M. Hossain, InTech, Croatia, 2011, p. 189.

[3] I. Mogi, K. Watanabe, ISIJ Int. 47, 585 (2007).

[4] I. Mogi, K. Watanabe, Magnetohydrodynamics 48, 251 (2012).

[5] I. Mogi, K. Watanabe, Chem. Lett. 41, 1439 (2012). 